\title{
Developmental imaging genetics: Challenges and promises for translational research
}

\author{
ESSI VIDING, ${ }^{a}$ DOUGLAS E. WILLIAMSON,${ }^{b}$ AND AHMAD R. HARIRI ${ }^{b}$ \\ ${ }^{a}$ University College London; and ${ }^{b}$ University of Pittsburgh School of Medicine
}

\begin{abstract}
Advances in molecular biology, neuroimaging, genetic epidemiology, and developmental psychopathology have provided a unique opportunity to explore the interplay of genes, brain, and behavior within a translational research framework. Herein, we begin by outlining an experimental strategy by which genetic effects on brain function can be explored using neuroimaging, namely, imaging genetics. We next describe some major findings in imaging genetics to highlight the effectiveness of this strategy for delineating biological pathways and mechanisms by which individual differences in brain function emerge and potentially bias behavior and risk for psychiatric illness. We then discuss the importance of applying imaging genetics to the study of psychopathology within a developmental framework. By beginning to move toward a systems-level approach to understanding pathways to behavioral outcomes as they are expressed across development, it is anticipated that we will move closer to understanding the complexities of the specific mechanisms involved in the etiology of psychiatric disease. Despite the numerous challenges that lie ahead, we believe that developmental imaging genetics has potential to yield highly informative results that will ultimately translate into public health benefits. We attempt to set out guidelines and provide exemplars that may help in designing fruitful translational research applications that incorporate a developmental imaging genetics strategy.
\end{abstract}

Recent advances in both molecular genetics and human neuroimaging have begun to provide the tools necessary to explore how individual differences in complex cognitive and emotional behaviors emerge and how such differences may confer vulnerability to psychopathology. With publication of the reference human genome sequence (Lander et al., 2001; Venter et al., 2001), a major effort is under-

This research was supported in part by funding from the United Kingdom National Programme on Forensic Mental Health Research and Development (to E.V.), the Medical Research Council Grant G0401170 (to E.V.), the National Institute of Mental Health Grants K01-MH001957 (to D.E.W.) and K01-MH072837 (to A.R.H.), as well as an NARSAD Young Investigator Award (to A.R.H.).

Address correspondence and reprint requests to: Ahmad R. Hariri, Developmental Imaging Genetics Program, Department of Psychiatry, University of Pittsburgh School of Medicine, Western Psychiatric Institute and Clinic, 3811 O'Hara Street, Room E-729, Pittsburgh, PA 15213-2593; E-mail: harirar@upmc.edu. way to exhaustively identify common variations in this sequence that impact on gene function (i.e., functional polymorphisms) and subsequently to understand how such functional variations alter human biology. Because approximately $70 \%$ of all genes are expressed in the brain, many of these functional polymorphisms will influence how the brain processes information and, as a consequence, regulates both cognitive and affective behaviors. Human neuroimaging (e.g., functional magnetic resonance imaging [fMRI], EEG/magnetic encephalography [MEG], positron emission tomography), because of its capacity to assay detailed brain structure and function within individuals, has unique potential as a tool for characterizing functional genetics in neural circuitry. The goals of this paper are to (a) describe the conceptual basis for imaging genetics; (b) outline some major findings in imaging genetics to highlight the effectiveness of this strategy in delineating 
biological pathways and mechanisms by which individual differences in brain function emerge and potentially bias risk for psychiatric illness; (c) discuss the importance of applying the imaging genetics framework to study development and the emergence of psychopathology; (d) illustrate designs of developmental imaging genetics studies; and (e) summarize the anticipated short- and long-term rewards of this program of research, with an emphasis on the translational impact of this approach.

\section{Conceptual Basis of Imaging Genetics}

Genes have unparalleled potential impact on all levels of biology. In the context of disease states, particularly behavioral disorders, genes represent the cornerstone of mechanisms that either directly or in concert with environmental events ultimately result in disease. Moreover, genes offer the potential to identify at-risk individuals and biological pathways for the development of new treatments. Although most human behaviors cannot be explained by genes alone, and certainly much variance in aspects of brain information processing will not be genetically determined directly, it is anticipated that variations in genetic sequence that impact gene function will contribute an appreciable amount of variance to these resultant complex behavioral phenomena. This conclusion is implicit in the results of studies of twins that have revealed heritabilities ranging from 40 to $70 \%$ for various aspects of cognition, temperament, and personality (Plomin, Owen, \& McGuffin, 1994). In the case of psychiatric illness, genes appear to be the only consistent risk factors that have been identified across populations, and the majority of susceptibility for major psychiatric disorders is accounted for by inheritance (Moldin \& Gottesman, 1997).

Traditionally, the impact of genetic polymorphisms on human behavior has been directly examined using clinical evaluations, personality questionnaires, and neuropsychological batteries. Genetic epidemiological investigations have directly examined the relationship between specific genetic polymorphisms and behaviors, and have reported equivocal results (Malhotra \& Goldman, 1999). This is not surprising for at least two reasons. First, there is considerable individual variability in dimensions of observable behavior as well as subjectivity in the assessment of behavior necessitating very large samples, often exceeding several hundred subjects, to identify even small gene effects (Glatt \& Freimer, 2002). Moreover, it is apparent that there are etiological subgroups within any given disease that obscure effects at the broader group level. Second, and perhaps most importantly, the effects of genes are not expressed directly at the level of behavior. As discussed in detail below, gene effects on behavior are mediated by their molecular and cellular effects on information processing in brain. Thus, examining gene effects on brain represents a critical step in understanding their ultimate contribution to variability in behavior.

Because genes are directly involved in the development and function of brain regions subserving specific cognitive and emotional processes, functional polymorphisms in genes may be strongly related to the function of these specific neural systems, and in turn, mediate/ moderate their involvement in behavioral outcomes. This is the underlying assumption of our investigations examining the relation between genes and neural systems, what we initially called, "imaging genomics" (Hariri \& Weinberger, 2003b), and more recently describe as "imaging genetics" (Hariri, Drabant, \& Weinberger, 2006), because this approach is utilized to explore variation in specific genes and not the genome broadly. The potential for marked differences at the neurobiological level underscores the need for a direct assay of brain function. Accordingly, imaging genetics within the context of a "candidate gene association approach" provides an ideal opportunity to further our understanding of biological mechanisms potentially contributing to individual differences in behavior and personality. Moreover, imaging genetics provides a unique tool with which to explore and evaluate the functional impact of brain-relevant genetic polymorphisms with the potential to understand their impact on behavior. Of course, the relevance of imaging genetics findings for disease vulnerability will only be made once the vari- 
ants under study are further associated with disease risk directly or if their impact on brain function is manifest (or even exaggerated) in the diseases of interest.

Neuroimaging techniques, especially those that are noninvasive like MRI and EEG/ MEG, typically require no more than a few minutes of subject participation to acquire substantial data sets, reflecting the acquisition of many hundreds of repeated measures of brain structure or function within a single subject. The efficiency of these techniques allows for the ability to investigate the specificity of gene effects by examining their influence on multiple functional systems (e.g., prefrontal, striatal, limbic) in a single subject in one experimental session. This capacity to rapidly assay differences in brain structure and information processing with enhanced power and sensitivity places neuroimaging at the forefront of available tools for the in vivo study of functional genetic variation.

The protocol for imaging genetics typically involves first identifying a meaningful variation in the DNA sequence within a candidate gene. For the variant to be meaningful, it should have an impact at the molecular and cellular level in gene or protein function (i.e., be a functional variant), and the distribution of such effects at the level of brain systems involved in specific forms of information processing should be predictable. For example, a genetic variation in the gene for the serotonin transporter that impacts the availability of synaptic serotonin would be expected to affect amygdala function because serotonin is important in amygdala physiology (see below). Alternatively, recent imaging genetics studies have taken the lead in exploring the functionality of candidate variants by first describing in vivo effects at the level of brain systems (Brown et al., 2005). As such, imaging genetics can provide the initial impetus for further characterization of molecular and functional effects of specific candidate genes in brain systems thought to be involved in behavior. In this manner, the contributions of abnormalities in these systems to complex behaviors and emergent phenomena, possibly including psychiatric syndromes, can then be understood from the perspective of their biological origins.

\section{Selected Overview of Imaging Genetics Findings}

Converging evidence from animal and human studies has revealed that serotonin (5-hydroxytryptamine [5-HT]) is a critical neuromodulator in the generation and regulation of emotional behavior (Lucki, 1998). Serotonergic neurotransmission has also been an efficacious target for the pharmacological treatment of mood disorders including depression, obsessive-compulsive disorder, anxiety, and panic (Blier \& de Montigny, 1999). Moreover, genetic variation in several key 5-HT subsystems, presumably resulting in altered central serotonergic tone and neurotransmission, has been associated with various aspects of personality and temperament (Munafo, Clark, \& Flint, 2005; Schinka, Busch, \& Robichaux-Keene, 2004; Sen, Burmeister, \& Ghosh, 2004), as well as susceptibility to affective illness (Murphy et al., 1998; Reif \& Lesch, 2003). However, enthusiasm for the potential of such genetic variation to affect behaviors and especially disease liability has been tempered by weak, inconsistent, and failed attempts at replication of specific associations with psychiatric syndromes (Glatt \& Freimer, 2002).

The inability to substantiate such relationships through consistent replication in independent cohorts may simply reflect methodological issues such as inadequate control for population stratification, insufficient power, and/or inconsistency in the methods applied. Alternatively, and perhaps more importantly, such inconsistency may reflect the underlying biological nature of the relationship between allelic variants in serotonin genes, each of presumably small effect, and observable behaviors in the domain of mood and emotion that typically reflect complex functional interactions and emergent phenomena. Given that the biological impact of variation in a gene traverses an increasingly divergent path from cells to neural systems to behavior, the response of brain regions subserving emotional processes in humans (e.g., amygdala, hippocampus, prefrontal cortex, anterior cingulate gyrus) represents a critical first step in their impact on behavior. Thus, functional polymor- 
phisms in 5-HT genes may be strongly related to the integrity of these underlying neural systems and mediate/moderate their ultimate effect on behavior (Hariri \& Weinberger, 2003a).

The 5-HT transporter (5-HTT) plays an important role in serotonergic neurotransmission by facilitating reuptake of 5-HT from the synaptic cleft. In 1996, a relatively common polymorphism was identified in the human 5-HTT gene located on chromosome 17q11.1q12 (Heils et al., 1996). The polymorphism is a variable repeat sequence in the promoter region (5-HTTLPR) resulting in two common alleles: the short (S) variant comprising 14 copies of a 20-23 base pair repeat unit, and the long (L) variant comprising 16 copies. In populations of European ancestry, the frequency of the $\mathrm{S}$ allele is approximately 0.40 , and the genotype frequencies are in HardyWeinberg equilibrium $(\mathrm{L} / \mathrm{L}=0.36, \mathrm{~L} / \mathrm{S}=$ $0.48, \mathrm{~S} / \mathrm{S}=0.16)$. These relative allele frequencies, however, can vary substantially across populations (Gelernter, Kranzler, \& Cubells, 1997).

Following the identification of this polymorphism, Lesch et al. (1996) demonstrated in vitro that the 5-HTTLPR alters both gene transcription and level of 5-HTT function. Cultured human lymphoblast cell lines homozygous for the long allele have higher concentrations of 5-HTT mRNA and express nearly twofold greater 5-HT reuptake in comparison to cells possessing either one or two copies of the short allele. Subsequently, both in vivo imaging measures of radioligand binding to 5-HTT (Heinz et al., 2000) and postmortem calculation of 5-HTT density (Little et al., 1998) in humans reported nearly identical reductions in 5-HTT binding levels associated with the short allele as observed in vitro (but see Patkar et al., 2004; Shioe et al., 2003; van Dyck et al., 2004). These data are consistent with $\beta$-CIT SPECT studies in humans and nonhuman primates reporting an inverse relationship between 5-HTT availability and CSF concentrations of 5-hydroxyindoleacetic acid (5-HIAA), a 5-HT metabolite (Heinz et al., 1998, 2002), and indicate that the 5-HTTLPR is functional and impacts on serotonergic neurotransmission.
In their initial study, Lesch and colleagues also demonstrated that individuals carrying the short allele are slightly more likely to display abnormal levels of anxiety in comparison to L/L homozygotes (Lesch et al., 1996). Since their original report, others have confirmed the association between the 5-HTTLPR short allele and heightened anxiety (Du, Bakish, \& Hrdina, 2000; Katsuragi et al., 1999; Mazzanti et al., 1998; Melke et al., 2001), and have also demonstrated that individuals possessing the short allele more readily acquire conditioned fear responses (Garpenstrand, Annas, Ekblom, Oreland, \& Fredrikson, 2001) and develop affective illness (Lesch \& Mossner, 1998) in comparison to those homozygous for the long allele. Recent studies utilizing pharmacological challenge paradigms of the 5-HT system suggest that these differences in affect, mood, and temperament may reflect 5-HTTLPR driven variation in 5-HTT expression and subsequent changes in synaptic concentrations of 5-HT (Moreno et al., 2002; Neumeister et al., 2002; Whale, Clifford, \& Cowen, 2000). Furthermore, reduced 5-HTT availability, as putatively indexed by the 5-HTTLPR short allele, has been associated with mood disturbances including major depression (Caspi et al., 2003; Malison et al., 1998) and the severity of depression and anxiety in various psychiatric disorders (Eggers et al., 2003; Heinz et al., 2002; Willeit et al., 2000). Intriguingly, it appears that exposure to stressful life events moderates the impact of the 5-HTTLPR for the development of depression (Caspi et al., 2003).

The amygdala is a central brain structure in the generation of both normal and pathological emotional behavior, especially fear (LeDoux, 2000). Furthermore, the amygdala is densely innervated by serotonergic neurons and 5-HT receptors are abundant throughout amygdala subnuclei (Azmitia \& Gannon, 1986; Sadikot \& Parent, 1990; Smith, Daunais, Nader, \& Porrino, 1999). Thus, the activity of this subcortical region may be uniquely sensitive to alterations in serotonergic neurotransmission, and any resulting variability in amygdala excitability is likely to contribute to individual differences in emergent phenomena such as mood and temperament. How- 
ever, it is essential to appreciate the importance of a distributed and interconnected network of cortical and subcortical brain regions for the generation, integration, and modulation of emotional behavior. Results from a series of landmark imaging studies (Beauregard, Levesque, \& Bourgouin, 2001; Hariri, Bookheimer, \& Mazziotta, 2000; Keightley et al., 2003; Lange et al., 2003; Nakamura et al., 1998; Narumoto et al., 2000) suggest that the dynamic interactions of the amygdala and prefrontal cortex may be critical in regulating emotional behavior (Hariri, Mattay, Tessitore, Fera, \& Weinberger, 2003).

Although the potential influence of genetic variation in 5-HTT function on human mood and temperament was bolstered by subsequent studies demonstrating increased anxiety-like behavior and abnormal fear conditioning in 5-HTT knockout mice (Holmes, Lit, Murphy, Gold, \& Crawley, 2003), the underlying neurobiological correlates of this functional relationship remain unknown. Because the physiologic response of the amygdala during the processing of fearful or threatening stimuli temporally precedes the subjective experience of emotionality, the 5-HTTLPR may have a more obvious impact at the level of amygdala biology.

In 2002, our research group at the National Institutes of Mental Health utilized an imaging genetics strategy with fMRI to directly explore the neural basis of the apparent relationship between the 5-HTTLPR and emotional behavior (Hariri, Mattay, Tessitore, Kolachana, et al., 2002). Specifically, we hypothesized that 5-HTTLPR short allele carriers, who presumably have relatively lower 5-HTT function and higher synaptic concentrations of 5-HT (analogous to the 5-HTT knockout mice) and have been reported to be more anxious and fearful, would exhibit greater amygdala activity in response to fearful or threatening stimuli than those homozygous for the long allele, who presumably have lower levels of synaptic 5-HT and have been reported to be less anxious and fearful (analogous to the contrasting wild-type mice).

In our initial study, subjects from two independent cohorts $(n=14$ in each) were divided into equal groups based on their 5-HTTLPR genotype, with the groups matched for age, gender, IQ and task performance. During scanning, the subjects performed a simple perceptual processing task involving the matching of fearful and angry human facial expressions. Importantly, this task has been effective at consistently engaging the amygdala across multiple subject populations and experimental paradigms (Hariri et al., 2000; Hariri, Mattay, Tessitore, Fera, et al.., 2002; Hariri, Tessitore, Mattay, Fera, \& Weinberger, 2002; Tessitore et al., 2002). Consistent with our hypothesis, we found that subjects carrying the less efficient 5-HTTLPR short allele exhibited significantly increased amygdala activity in comparison with subjects homozygous for the L allele (Hariri, Mattay, Tessitore, Kolachana, et al., 2002). In fact, the difference in amygdala activity between 5-HTTLPR genotype groups in this study was nearly fivefold, accounting for $20 \%$ of the total variance in the amygdala response. This initial finding suggested that the increased anxiety and fearfulness associated with individuals possessing the 5-HTTLPR S allele may reflect the hyperresponsiveness of their amygdala to relevant environmental stimuli.

Recently, five independent functional imaging studies have reported identical 5-HTTLPR S allele driven amygdala hyperreactivity in cohorts of healthy German (Heinz et al., 2005), Italian (Bertolino et al., 2005) and American (Canli et al., 2005) adult volunteers, as well as Dutch patients with social phobia (Furmark et al., 2004) and German patients with panic disorder (Domschke et al., 2005). Moreover, we have also replicated our initial finding of 5-HTTLPR S effects on amygdala reactivity in a large, independent cohort of adult volunteers $(n=92)$ who were carefully screened to exclude individuals with a history of psychiatric illness or treatment. This large sample also allowed for the exploration of both gender-specific and S allele load effects on amygdala function and, in turn, dimensions of temperament associated with depression and anxiety (see below).

Specifically, we again observed that 5-HTTLPR S allele carriers exhibited significantly increased right amygdala activation in response to our fMRI challenge paradigm 
(Hariri et al., 2005). In addition, our latest data revealed that 5-HTTLPR S allele driven amygdala hyperresponsivity is equally pronounced in both sexes and independent of $\mathrm{S}$ allele load. The equivalent effect of one or two $\mathrm{S}$ alleles on amygdala function is consistent with the original observations of Lesch et al. (1996) on the influence of the 5-HTTLPR on in vitro gene transcription efficiency and subsequent 5-HTT availability. The absence of sex differences suggests that the increased prevalence of mood disorders in females may be related to factors other than the direct risk effect of the 5-HTTLPR S allele.

A third study from the NIMH cohort further captured the dynamic effects of the 5-HTTLPR on genes, brain, and behavior by examining effects on brain structure and corticolimbic functional connectivity (Pezawas et al., 2005). Here, we used a multimodal neuroimaging strategy to identify mechanisms on the level of neural systems contributing to behavioral and, potentially, clinical effects associated with 5-HTTLPR. We used voxel-based structural MRI techniques in a large sample $(N=114)$ to test for genetic association with the anatomical development of limbic circuitry, as might be expected from neurodevelopmental studies of animals with altered 5-HT function (Gaspar, 2004). We found that, in comparison to the LL genotype subjects, $\mathrm{S}$ allele carriers showed significantly reduced gray matter volume of the perigenual anterior cingulate cortex (pACC) and amygdala. Moreover, there was a positive correlation ("structural covariation") between amygdala and pACC volume, and S carriers showed significantly lower structural covariation between amygdala and subgenual anterior cingulate than LL individuals. This suggests that $\mathrm{pACC}$ and amygdala represent a functional circuit, the morphological development of which is modulated by genetic variation in the serotonergic system.

We next explored the impact of these observed structural effects on the functional interactions of the amygdala and pACC in our fMRI data set. Independent of 5-HTTLPR genotype status, we found that the amygdala and pACC were significantly functionally connected. Two distinct regions of functional con- nectivity were identified within the pACC, a positive coupling between the amygdala and the subgenual cingulate, and a negative coupling between the amygdala and the supragenual cingulate. This pattern of functional connectivity is consistent with anatomical tracing studies in nonhuman primates that have defined a feedback circuit from amygdala to rostral cingulate and then from dorsal cingulate back to amygdala. These intrinsic cingulate regions also showed strong positive connectivity with each other, suggesting that the corticolimbic feedback loop is closed via local processing within the cingulate cortex. This intrinsic cingulate connection also is consistent with anatomical studies in nonhuman primates. Remarkably, 5-HTTLPR S allele carriers showed a significant reduction of amygdala-pACC functional connectivity in comparison to LL homozygotes. This difference was most pronounced in the coupling of the amygdala and subgenual ACC. These findings suggest that a disruption of this amygdalapACC feedback circuitry could underlie the earlier observation of increased amygdala activity in $\mathrm{S}$ carriers during the processing of biologically salient stimuli (Hariri et al., 2005; Hariri, Mattay, Tessitore, Kolachana, et al., 2002). More specifically, the data suggest that the over activation of the amygdala associated with the 5-HTTLPR short allele may reflect more a relative failure of regulation of the amygdala response, than an abnormal primary response per se. Taken together, these data show that 5-HTTLPR genotype affects the structure and putative wiring of a core region within the limbic system thought to be crucial for anxiety-related temperamental traits and depression (Mayberg, 2003a, 2003b; Phillips, Drevets, Rauch, \& Lane, 2003a, 2003b). These findings thus suggest a causal mechanism linking developmental alterations in 5-HTdependent neuronal pathways to impaired interactions in a regulatory network that has been related to emotional reactivity.

The collective results of these imaging genetics studies reveal that the 5-HTTLPR S allele has a robust effect on human amygdala structure and function as well as the functional interactions of corticolimbic circuitry implicated in both normal and pathological 
mood states. Importantly, the absence of group differences in age, gender, IQ and ethnicity in each of these studies indicates that the observed effects are not likely due to a bias resulting from population stratification. Rather, the data suggest that heritable variation in 5-HT signaling associated with the 5-HTTLPR results in structural alterations of the amygdala and pACC, accompanied by biased amygdala reactivity and functional coupling with pACC in response to salient environmental cues. Furthermore, the emergence of these effects in samples of ethnically matched volunteers carefully screened to exclude any lifetime history of psychiatric illness or treatment argues that they represent genetically determined biological traits that are not altered by the presence of a psychiatric illness.

In contrast to the striking imaging genetics findings of 5-HTTLPR short allele effects on amygdala reactivity and limbic circuitry dynamics, initial attempts to link these effects on brain function with measures of emergent behavioral phenomena, namely, the personality trait of harm avoidance, have failed to detect any significant direct relationships. Specifically, in both our initial (Hariri, Mattay, Tessitore, Kolachana, et al., 2002) and replication studies (Hariri et al., 2005) we did not find any significant 5-HTTLPR genotype association with subjective behavioral measures of anxiety-like or fear-related traits as indexed by the harm avoidance (HA) component of the Tridimensional Personality Questionnaire, a putative personality measure related to trait anxiety and 5-HT function (Cloninger, 1986; Cloninger, Svrakic, \& Przybeck, 1993). This failure to find a behavioral association is not surprising given the relatively small sample sizes of each study and thus limited power to detect likely small (e.g., 1-5\%) genetically mediated differences in behavior as well as the theses of this paper: that genes do not directly predict behavior and their effect on behavior is mediated/moderated by their effects on distinct brain circuitry.

However, to our surprise, there was an absence of any predictive relationship between amygdala reactivity and HA in our initial studies. Given the critical role for the amygdala in detecting potential environmental threat and harnessing available resources for appropriate reactions, one might expect that its reactivity to such stimuli would predict individual differences in a temperamental trait such as HA. But, a convergence of evidence from animal and human studies clearly demonstrates that emotional behaviors, especially those as complex as HA, are likely influenced by a densely interconnected and distributed cortical and subcortical circuitry, of which the amygdala is only one component. Thus, we were compelled to examine the relationship between HA and the observed 5-HTTLPR effects on the functional connectivity of the amygdala and pACC. We reasoned that if functional uncoupling of the amygdala-pACC affective circuit underlies reported associations of 5-HTTLPR with emotional phenotypes, functional connectivity indices between these regions should predict normal variation in temperamental trait measures related to anxiety and depression such is HA. These analyses revealed a striking pattern wherein nearly $30 \%$ of the variance in HA scores was predicted by our measure of amygdala-pACC functional connectivity (Pezawas et al., 2005). Consistent with our previous studies (Hariri et al., 2005; Hariri, Mattay, Tessitore, Kolachana, et al., 2002), functional (or structural) measures of single brain regions (i.e., amygdala or pACC) were of no predictive value. Thus, 5-HTTLPR-mediated corticolimbic functional connectivity alterations are manifested in anxiety-related temperamental traits, possibly reflecting inadequate regulation and integration of amygdala mediated arousal, leading to an increased vulnerability for persistent negative affect and eventually depression in the context of accumulating environmental adversity. Although investigations of localized structural and functional abnormalities have provided insights about depression, our data underscore the importance of studying genetic mechanisms of complex brain disorders at the level of dynamically interacting neural systems. We suggest that such relationships capture more proximally the functional consequences of neurodevelopmental processes altering circuitry function implicated in human temperament and psychiatric disorders. 


\section{Importance of Imaging Genetics for Understanding Development and Developmental Psychopathology}

It is important to emphasize that the 5-HTTLPR S allele effect on amygdala structure, reactivity, and connectivity in our studies as well as those by Heinz et al., Bertolino et al., and Canli et al. exist in samples of healthy adult volunteers with no history of affective or other psychiatric disorders. On the one hand, this is consistent with a recent fMRI study reporting that, although amygdala hyperexcitability reflects a stable, heritable trait associated with inhibited behavior, it does not by itself predict the development of affective disorders (Schwartz, Wright, Shin, Kagan, \& Rauch, 2003). On the other hand, more and more evidence is accumulating which indicates that the majority of psychopathology is rooted early in life first emerging during childhood and adolescence (e.g., KimCohen et al., 2003). Thus, it is possible that the relevance of 5-HTTLPR S allele effects on corticolimbic brain circuitry will be more manifest during the development of individuals predisposed to psychopathology. Moreover, it is likely that exposure to environmental stressors impacts this gene-brain pathway, which in turn, increases one's risk to develop psychopathology. The hallmark study of Caspi et al. (2003) and subsequent replication studies (Eley et al., 2004; Kaufman et al., 2004; Kendler et al., 2005) suggest that the existence of significant stressors in the environment of individuals carrying the 5-HTTLPR S allele is necessary to further tip the balance towards the development of psychopathology. Similarly, abnormal social behavior (Champoux et al., 2002) and 5-HT metabolism (Bennett et al., 2002) have been reported in rhesus macaques with the 5-HTTLPR S allele homo$\log$, but only in peer-reared, and thus environmentally stressed, individuals. Emerging data from studies of the 5-HTT knockout mouse implicate similar early developmental phenomena interacting with genetically driven variation in 5-HT in shaping the neurobiological landscape contributing to emotional behaviors (Ansorge, Zhou, Lira, Hen, \& Gingrich, 2004; Esaki et al., 2005; Holmes \& Hariri,
2003). It is pertinent to note that in many of these examples the genetic vulnerability has manifested as a consequence of environmental stressors that have occurred early in development.

This shift from normal to pathological behaviors, and when during the lifespan this shift occurs may reflect the effects of cumulative environmental stress on brain regions, most notably the prefrontal cortex, critical in the regulation of amygdala activity (Hariri et al., 2003; Keightley et al., 2003; Rosenkranz, Moore, \& Grace, 2003). For example, repeated exposure to environmental insults before the maturation of relatively late developing prefrontal regulatory circuits (Lewis, 1997) may result in further biased amygdala drive in $\mathrm{S}$ allele carriers. Such relative hyperamygdala and hypoprefrontal activity has been documented in affective disorders (Phillips et al., 2003b; Siegle, Steinhauer, Thase, Stenger, \& Carter, 2002) and thus, may represent a critical pathway or predictive biological marker for the future development of psychopathology.

Recent imaging studies (Heinz et al., 2005; Pezawas et al., 2005) demonstrating altered functional coupling of the amygdala and medial prefrontal cortex during affective processing in adult healthy $\mathrm{S}$ allele carriers underscore that complex dynamic interactions of the amygdala and prefrontal cortex may be critical for normal behavioral responses in individuals possessing this risk allele. These results suggest that individual differences in indices of complex, emergent behaviors, such as harm avoidance, reflect the effects of genetic variation on a distributed brain system involved in not only mediating physiologic and behavioral arousal (e.g., amygdala) but also regulating and integrating this arousal in the service of adaptive responses to environmental challenges (e.g., prefrontal cortex)

As Rutter and colleagues suggest, some environmental adversity during early development (and likely throughout one's lifetime) is necessary to enable an individual to cope with the stresses and challenges of everyday life (Rutter, Kim-Cohen, \& Maughan, 2006; Rutter, Moffitt, \& Caspi, 2006). However, some experiences are beyond the range of normative environmental stress, and continually en- 
countering such experiences may, in the long run, compromise an individual's ability to respond to environmental events in an adaptive way. It is likely that genetic makeup influences the "fingerprint" of how an individual reacts to stress as well as determines their resiliency (i.e., adaptive functioning in the face of adversity) to chronic stressors. There have been calls to incorporate a genetic/biological perspective to resiliency research that has predominantly consisted of psychosocial investigations (Curtis \& Cicchetti, 2003). The biological fingerprint of resiliency is likely to vary by disorder as well as a function of environmental circumstances (Luthar, Cicchetti, $\&$ Becker, 2000). Developmental imaging genetics represents one unique approach by which resiliency can be investigated within a translational research paradigm.

As mentioned above, adult psychiatric disorders rarely arise de novo, without any warning of childhood problems including fullblown episodes of psychopathology (KimCohen et al., 2003). Some psychiatric disorders may lie dormant until a crucial point in development or until a critical environmental stressor is present to precipitate their onset. As such, an individual may have a vulnerability to a disorder from birth, but this vulnerability may only manifest later in life. Depression and schizophrenia are two disorders that traditionally have not been diagnosed before adolescence or early adulthood, although sufficient evidence has accumulated to suggest that aspects of each originate in early development (Cicchetti \& Cannon, 1999; Kim-Cohen et al., 2003). Other adult disorders represent the eventual manifestation of a behavioral difficulty visible since early childhood. Antisocial personality disorder is an example where one of the diagnostic criteria includes evidence of childhood conduct problems. In all cases, it is vital to understand the developmental course of psychopathologies that inform our efforts to understand the etiology, progression, and treatment of psychiatric disease. As part of such a broader developmental research program, imaging genetics has great potential yield in identifying component etiologic processes of psychopathology as well as the emergence of normal individual differences in behavior.
Accordingly, we speculate that adult imaging genetics findings represent windows into systems whose current structure and function resulted from developmental alterations during unique periods of plasticity, long before the physiological associations were captured via neuroimaging in adulthood (i.e., they represent "ghosts in the machine").

One avenue of potential fruitful research into these developing systems is the use of longitudinal studies beginning in childhood. This approach represents an ideal way to examine the impact of genetic and environmental effects on the developing neural circuitry supporting behavior and conferring risk for psychopathology. Such an approach will allow for the determination of genetically driven variation on structural and functional brain development during windows of time that reflect critical maturational processes (e.g., myelination, synaptic pruning). Longitudinal studies in at-risk populations prior to the development of psychopathology will further allow for a more accurate and rigorous assessment and recording of environmental events and experiences as well as their interplay with genetically mediated risks. Moreover, a longitudinal approach will facilitate charting the behavioral consequences, including risk for psychopathology, of such genetically and environmentally driven variation in brain structure and function. Such studies will serve not only to collect important baseline structural and functional data during critical stages of development but also to enable us to disentangle how genetic and environmental factors converge during development to bias brain systems and, in turn, mediate risk for psychopathology.

With regard to development in general, although age is broadly considered as an index of development it may also be one of the most imprecise as it simultaneously serves as an index of physical maturation, hormonal state, cognitive level, social circumstances, and life experiences (Rutter, Kim-Cohen, et al., 2006). It is therefore crucial to attempt to disentangle which of these features associated with age is likely to have an influence with respect to the development of psychopathology. For some behaviors, this endeavor will be made easier by a rich research base that can identify spe- 
cific age-related variables crucial for understanding the target developmental change and its relevance in moderating risk or resiliency for psychopathology. For other phenomena, and often for brain function in particular, one will not have the luxury of a rich empirical database and will have to start with carefully formulated biologically constrained hypotheses about genetic influences on normal and abnormal brain function.

There are, of course, some limitations to this experimental strategy. For example, imaging genetics has only been used to observe the effects of a few polymorphic genes (or large chromosomal deletions; e.g., MeyerLindenberg et al., 2005) on brain structure and function in humans. However, a number of nonvarying genes are likely to be very important in initiating developmental cascades that have profound impact on brain structure/ function and ultimately behavior. Moreover, our understanding of the timing and biological impact of gene expression is conceptually simplistic and the interacting influences of other genes as well as environmental factors on the temporal and spatial patterns of gene expression is poorly described. At present, such effects are only tractable in animal models (Meaney \& Szyf, 2005). These types of genetic effects, which we cannot test for in developmental imaging genetics studies at the present time, are likely to provide "background noise" in our assessments of specific candidate genes and gene-environment interactions.

Despite the fact that longitudinal developmental imaging data will naturally require concerted and protracted efforts and is unable to study the effects of nonpolymorphic genes on human brain development, there remains tremendous value in developmental imaging genetics "here and now." For example, it is of interest to investigate whether the 5-HTTLPR effects on the functional connectivity of the amygdala and pACC can be seen prior to the maturation of prefrontal cortex in adolescence, a period of increased risk for mood disorders, and influence temperament in similar ways as in adults. Cross-sectional developmental data would be adequate in initially addressing this question.

\section{Developmental Imaging Genetics: Ongoing Investigations}

We will briefly describe two of our ongoing developmental studies in an effort to illustrate potential applications of translational research that incorporates a developmental imaging genetics approach to examine the biological pathways to normal and pathological behavior. These two transcontinental studies are aimed at (a) identifying pathways to antisocial behavior ( $\mathrm{AB}$ ) and (b) elucidating an affective pathway to the development of alcohol use disorders (AUDs) emerging in adolescence.

\section{Development of antisocial behavior}

Children with early-onset antisocial behavior are at risk to develop chronic persistent antisocial behavior during the course of their lifetime (Eley, Lichtenstein, \& Moffitt, 2003). Elucidating different developmental pathways to persistent antisocial behavior is of major importance as individuals with earlyonset antisocial behavior are 10 times more costly to society compared to controls (Bailey, 2002; Scott, Knapp, Henderson, \& Maughan, 2001). In this context, the aim of a developmental imaging genetics approach would be to increase our understanding of both the genetic and neurobiological mechanisms of vulnerability in antisocial children and ultimately to identify pathways for the developmental of extreme behavioral problems.

One risk factor that appears to have predictive importance for life-course persistent antisocial behavior is the presence of callousunemotional (CU) traits (e.g., lack of guilt and empathy). Adult psychopaths (individuals with severe overt antisocial behavior coupled with CU traits) are chronic, versatile, and serious offenders who have early emerging behavioral problems (Hare, Hart, \& Harpur, 1991). Children with early-onset antisocial behavior coupled with CU traits are more likely to persist with antisocial conduct and commit more serious acts of antisocial behavior than their antisocial peers with nonelevated levels of CU traits (Frick, Cornell, Barry, Bodin, \& Dane, 2003). We recently demonstrated that CU traits delineate an etiologically distinct 
subgroup of children with early-onset antisocial behavior (Viding, Blair, Moffitt, \& Plomin, 2005) as indexed by high heritability estimates of $81 \%$ among the $\mathrm{AB}$ children with elevated levels of CU traits compared to only $30 \%$ among the $\mathrm{AB}$ children without elevated levels of CU traits (Viding et al., 2005). Given the level of the genetic contribution to $\mathrm{AB}$ with CU traits and the demonstrated impact of genetic polymorphisms on neural systems supporting affective behaviors (e.g., 5-HTTLPR effects on corticolimbic circuitry), we reasoned that there was a high likelihood that genetically driven biological systems contribute to the emergence of these traits.

In short, from our work we know that the $\mathrm{AB}+\mathrm{CU}$ subtype is likely to be under genetic mediation, as evidenced by high heritability and likely involves alterations in the corticolimbic circuitry mediating arousal and vigilance. As such, we reasoned that the selection of candidate genes on the basis of previous investigations (e.g., 5-HTTLPR) would potentially help to elucidate genetic moderation of alterations in this corticolimbic circuitry. Accordingly, we have undertaken a developmental imaging genetics project to (a) document whether preadolescent children with $\mathrm{AB}+\mathrm{CU}$ have distinct corticolimbic dysfunction, (b) whether this dysfunction is heritable, and (c) what specific genes (e.g., 5-HTTLPR) can be found to be associated with this dysfunction. Our investigation is being conducted in two steps. First, children with $\mathrm{AB}+\mathrm{CU}(N=12)$ are being compared to those who have $\mathrm{AB}$ without $\mathrm{CU}(N=12)$ and ability-matched controls $(N=12)$. This will enable us to determine whether there is unique brain dysfunction associated with $\mathrm{AB}+\mathrm{CU}$, rather than with $\mathrm{AB}$ in general (we are predicting that amygdala hyporeactivity to others' distress will be a unique marker for $\mathrm{CU}+\mathrm{AB}$ ). We will then recruit a larger sample of identical and nonidentical twins (132 twins in total), in concordant and discordant $\mathrm{AB}+\mathrm{CU}$ pairs, as well as control (unaffected) pairs. This will enable us to estimate the net contribution of genes generally as well as candidate polymorphisms specifically on brain activation differences between $\mathrm{AB}+\mathrm{CU}$ and control children (heritability). In this initial investigation, children aged 10 to 11 years are being assessed crosssectionally. However, we will follow these children longitudinally to see whether the pattern of amygdala reactivity will predict later antisocial behavior and whether this is moderated by $\mathrm{CU}$ traits as well as individual experiences with environmental stressors/precipitators.

\section{An affective pathway for the development of AUD}

AUD is a multifactorial disorder in which the environment interacts with genetic predisposition to produce the final level of risk (Schuckit, 1998). It has been shown that stress and affective responses to stress, including depression, influence alcohol drinking, and relapse (Kreek \& Koob, 1998). Although the relation between stress and alcohol drinking in humans (Pohorecky, 1991) and laboratory animals (Pohorecky, 1990) is complex, it is known that in some individuals increases in alcohol consumption are associated with stress. Although such stress-induced alcohol drinking and relapse behavior apparently have a significant genetic component (LaForge, Yuferov, \& Kreek, 2000; Wand et al., 2002), the neurobiological pathways underlying stress-induced alcohol drinking and relapse behavior are still obscure.

Adolescence is a crucial period for the development of affect regulation, affective disorders, and the onset of alcohol use. Although the relation between major depressive disorder (MDD) and AUD has been widely observed, there is currently little understanding of the shared etiology and diathesis of these two disorders, especially their initial emergence during adolescence. As part of our ongoing developmental work examining depression, we have recently investigated the emergence of AUD during adolescence among subjects initially studied in childhood (10-14 years old) and followed through early adulthood (16-20 years old). Analyses revealed that children with MDD as well as high familial loading for MDD had increased risk for developing AUD during adolescence in comparison with children with low familial loading for MDD. The risk for developing AUD did not differ between MDD and high familial-load 
children. In addition to group status effects, age at intake, gender, and follow-up months were all significant predictors of time to AUD. Our preliminary data strongly suggest that depression early in life and having high familial loading for depression significantly increase one's risk to develop AUD during adolescence. Coupled with the existing literature, these preliminary studies converge to highlight the need to understand the interplay between genes and environment on the development of neurobiological systems in adolescence as they impact one's risk to develop AUD.

As a result of our preliminary work, we have begun a project to disentangle several critical risk factors involved in the development of AUD this is moderated by preexisting risk for depression. Neuroimaging, neurobehavioral, and genetic measures are being used to achieve this goal and the study design has three phases. First, adolescents between the ages of 12 years and 14 years 11 months who have high $(n=150)$ and low $(n=150)$ familial loading for depression are being recruited. Initial assessments include measures of genes (primarily 5-HT subsystems like 5-HTTLPR), alcohol use, emotional behavior (depression/ negative affect, temperament), environmental stress (acute/chronic negative parent-child relationships), and imaging genetics assessments of brain function (amygdala reactivity and prefrontal regulatory control). A second phase of the proposed research project will consist of yearly follow-ups of all subjects to reassess behavior, environmental stress, and alcohol use outcomes. The final phase of our project will investigate the relationship of the development of AUD on neural circuitry central to the generation and regulation of arousal and affect.

\section{Translational aspects of developmental imaging genetics}

Although imaging genetics in and of itself provides a powerful new approach to the study of genes, brain, and behavior, its true potential will only be realized by aggressively expanding the scope and scale of the experimental protocols within a developmental framework, especially one that is focused on examining the developmental origins of behavior and disease. Although gene effects on brain function can be readily documented in samples of adults, the contributions of these genes acting in response to variable environmental pressures across development (when these systems are arguably most malleable) must be assessed to understand the biological pathways that bias behavior and risk for psychiatric illness. Combining these different strands of scientific enquiry (genetic, brain imaging, developmental, social) in a way that translates into public health benefits for affected individuals is in line with the current NIMH research direction (http://www.nimh.nih.gov/ strategic/strategicplanmenu.cfm). Translational research will be able to capitalize on developmental imaging genetics findings, and in the future, we will be able to document what constitute truly predictive markers for developmental outcome and disease progression as well as allow for the early identification of individuals at greater risk for emotional regulatory problems that can have long-term health related implications.

The short-term rewards of a developmental imaging genetics program are identifying the contributions of selected genetic, environmental, behavioral, and neurobiological factors for the emergence of individual differences in behavior and risk for disease. Although such short-term rewards can be realized with cross-sectional data, the long-term rewards of this approach, and thus the truly translational aspects of this work, will depend on longitudinal data. With such data we hope to gain greater understanding of how the genetic and environmental effects on circumscribed neuroanatomical circuits operate across development. Consequently, we can better document crucial time points for providing interventions targeted to specific neural processes. These interventions can also be tailored to take into account individual's genetic and environmental risk profiles. Thus, for some cases pharmacological intervention may be judged most appropriate, whereas for others, cognitive and behavioral approaches would be better suited to counter aberrant neural development. 


\section{Summary}

This article has outlined an experimental strategy by which genetic effects on brain function can be explored using neuroimaging, namely, imaging genetics. We discussed the effectiveness of this strategy for delineating biological pathways and mechanisms by which individual differences in brain function emerge and potentially bias behavior and risk for psychiatric illness. The main focus of this article was to highlight the importance of applying an imaging genetics framework to the study of psychopathology within a developmental

\section{References}

Ansorge, M. S., Zhou, M., Lira, A., Hen, R., \& Gingrich, J. A. (2004). Early-life blockade of the 5HT transporter alters emotional behavior in adult mice. Science, 306, 879-881.

Azmitia, E. C., \& Gannon, P. J. (1986). The primate serotonergic system: A review of human and animal studies and a report on macaca fascicularis. Advances in Neurology, 43, 407-468.

Bailey, S. (2002). Expert paper: Antisocial personality disorder: Children and adolescents. London: NHS National Programme on Forensic Mental Health Research and Development.

Beauregard, M., Levesque, J., \& Bourgouin, P. (2001). Neural correlates of conscious self-regulation of emotion. Journal of Neuroscience, 21, RC165.

Bennett, A. J., Lesch, K. P., Heils, A., Long, J. C., Lorenz, J. G., Shoaf, S. E., et al. (2002). Early experience and serotonin transporter gene variation interact to influence primate CNS function. Molecular Psychiatry, 7, 118-122.

Bertolino, A., Arciero, G., Rubino, V., Latorre, V., De Candia, M., Mazzola, V., et al. (2005). Variation of human amygdala response during threatening stimuli as a function of $5^{\prime}$ HTTLPR genotype and personality style. Biological Psychiatry, 57, 1517-1525.

Blier, P., \& de Montigny, C. (1999). Serotonin and druginduced therapeutic responses in major depression, obsessive-compulsive and panic disorders. Neuropsychopharmacology, 21(Suppl), 91S-98S.

Brown, S. M., Peet, E., Manuck, S. B., Williamson, D. E., Dahl, R. E., Ferrell, R. E., et al. (2005). A regulatory variant of the human tryptophan hydroxylase-2 gene biases amygdala reactivity. Molecular Psychiatry, 10, $884-888$.

Canli, T., Omura, K., Haas, B. W., Fallgatter, A., Constable, R. T., \& Lesch, K. P. (2005). Beyond affect: A role for genetic variation of the serotonin transporter in neural activation during a cognitive attention task. Proceedings of the National Academy of Sciences of the United States of America, 102, 12224-12229.

Caspi, A., Sugden, K., Moffitt, T. E., Taylor, A., Craig, I. W., Harrington, H., et al. (2003). Influence of life stress on depression: Moderation by a polymorphism in the 5-HTT gene. Science, 301, 386-389.

Champoux, M., Bennett, A., Shannon, C., Higley, J. D., framework, what we called developmental im aging genetics. We argued that by beginning to move toward a systems-level approach to understanding pathways to behavioral outcomes, as well as integrating the developmental angle, we will move closer to understanding the complexities of the specific mechanisms involved in the etiology of psychiatric disease. Numerous challenges lie ahead for developmental imaging genetics, but we believe that this approach has potential to yield highly informative results that will translate to public health benefits for people with psychiatric disorders.

Lesch, K. P., \& Suomi, S. J. (2002). Serotonin transporter gene polymorphism, differential early rearing, and behavior in rhesus monkey neonates. Molecular Psychiatry, 7, 1058-1063.

Cicchetti, D., \& Cannon, T. D. (1999). Neurodevelopmental processes in the ontogenesis and epigenesis of psychopathology. Developmental Psychopathology, 11, 375-393.

Cloninger, C. R. (1986). A unified biosocial theory of personality and its role in the development of anxiety states. Psychiatric Developments, 4, 167-226.

Cloninger, C. R., Svrakic, D. M., \& Przybeck, T. R. (1993) A psychobiological model of temperament and character. Archives in General Psychiatry, 50, 975-990.

Curtis, W. J., \& Cicchetti, D. (2003). Moving research on resilience into the 21 st century: Theoretical and methodological considerations in examining the biological contributors to resilience. Developmental Psychopathology, 15, 773-810.

Domschke, K., Braun, M., Ohrmann, P., Suslow, T., Kugel, H., Bauer, J., et al. (2005). Association of the functional-1019c/g 5-HT 1a polymorphism with prefrontal cortex and amygdala activation measured with $3 \mathrm{t}$ fMRI in panic disorder. International Journal of Neuropsychopharmacology, 9, 349-355.

Du, L., Bakish, D., \& Hrdina, P. D. (2000). Gender differences in association between serotonin transporter gene polymorphism and personality traits. Psychiatric Genetics, 10, 159-164.

Eggers, B., Hermann, W., Barthel, H., Sabri, O., Wagner, A., \& Hesse, S. (2003). The degree of depression in hamilton rating scale is correlated with the density of presynaptic serotonin transporters in 23 patients with Wilson's disease. Journal of Neurology, 250, 576-580.

Eley, T. C., Lichtenstein, P., \& Moffitt, T. E. (2003). A longitudinal behavioral genetic analysis of the etiology of aggressive and nonaggressive antisocial behavior. Developmental Psychopathology, 15, 383-402.

Eley, T. C., Sugden, K., Corsico, A., Gregory, A. M., Sham, P., McGuffin, P., et al. (2004). Geneenvironment interaction analysis of serotonin system markers with adolescent depression. Molecular Psychiatry, 9, 908-915.

Esaki, T., Cook, M., Shimoji, K., Murphy, D. L., Sokoloff, L., \& Holmes, A. (2005). Developmental disruption 
of serotonin transporter function impairs cerebral responses to whisker stimulation in mice. Proceedings of the National Academy of Sciences of the United States of America, 102, 5582-5587.

Frick, P. J., Cornell, A. H., Barry, C. T., Bodin, S. D., \& Dane, H. E. (2003). Callous-unemotional traits and conduct problems in the prediction of conduct problem severity, aggression, and self-report of delinquency. Journal of Abnormal Child Psychology, 31, 457-470.

Furmark, T., Tillfors, M., Garpenstrand, H., Marteinsdottir, I., Langstrom, B., Oreland, L., et al. (2004). Serotonin transporter polymorphism linked to amygdala excitability and symptom severity in patients with social phobia. Neuroscience Letters, 362, 1-4.

Garpenstrand, H., Annas, P., Ekblom, J., Oreland, L., \& Fredrikson, M. (2001). Human fear conditioning is related to dopaminergic and serotonergic biological markers. Behavioral Neuroscience, 115, 358-364.

Gaspar, P. (2004). Genetic models to understand how serotonin acts during development. Journal de la Société de Biologie, 198, 18-21.

Gelernter, J., Kranzler, H., \& Cubells, J. F. (1997). Serotonin transporter protein (slc6a4) allele and haplotype frequencies and linkage disequilibria in African- and European-American and Japanese populations and in alcohol-dependent subjects. Human Genetics, 101, 243-246.

Glatt, C. E., \& Freimer, N. B. (2002). Association analysis of candidate genes for neuropsychiatric disease: The perpetual campaign. Trends in Genetics, 18, 307-312.

Hare, R. D., Hart, S. D., \& Harpur, T. J. (1991). Psychopathy and the DSM-IV criteria for antisocial personality disorder. Journal of Abnormal Psychology, 100, 391-398.

Hariri, A. R., Bookheimer, S. Y., \& Mazziotta, J. C. (2000) Modulating emotional responses: Effects of a neocortical network on the limbic system. Neuroreport, 11, 43-48.

Hariri, A. R., Drabant, E. M., Munoz, K. E., Kolachana, B. S., Mattay, V. S., Egan, M. F., et al. (2005). A susceptibility gene for affective disorders and the response of the human amygdala. Archives in General Psychiatry, 62, 146-152.

Hariri, A. R., Drabant, E. M., \& Weinberger, D. R. (2006). Imaging genetics: Perspectives from studies of genetically driven variation in serotonin function and corticolimbic affective processing. Biological Psychiatry, 59, 888-897.

Hariri, A. R., Mattay, V. S., Tessitore, A., Fera, F., Smith, W. G., \& Weinberger, D. R. (2002). Dextroamphetamine modulates the response of the human amygdala. Neuropsychopharmacology, 27, 1036-1040.

Hariri, A. R., Mattay, V. S., Tessitore, A., Fera, F., \& Weinberger, D. R. (2003). Neocortical modulation of the amygdala response to fearful stimuli. Biological Psychiatry, 53, 494-501.

Hariri, A. R., Mattay, V. S., Tessitore, A., Kolachana, B., Fera, F., Goldman, D., et al. (2002). Serotonin transporter genetic variation and the response of the human amygdala. Science, 297, 400-403.

Hariri, A. R., Tessitore, A., Mattay, V. S., Fera, F., \& Weinberger, D. R. (2002). The amygdala response to emotional stimuli: A comparison of faces and scenes. Neuroimage, 17, 317-323.

Hariri, A. R., \& Weinberger, D. R. (2003a). Functional neuroimaging of genetic variation in serotonergic neurotransmission. Genes, Brain and Behavior, 2, 314-349.
Hariri, A. R., \& Weinberger, D. R. (2003b). Imaging genomics. British Medical Bulletin, 65, 259-270.

Heils, A., Teufel, A., Petri, S., Stober, G., Riederer, P., Bengel, D., et al. (1996). Allelic variation of human serotonin transporter gene expression. Journal of Neurochemistry, 66, 2621-2624.

Heinz, A., Braus, D. F., Smolka, M. N., Wrase, J., Puls, I., Hermann, D., et al. (2005). Amygdala-prefrontal coupling depends on a genetic variation of the serotonin transporter. Nature Neuroscience, 8, 20-21.

Heinz, A., Higley, J. D., Gorey, J. G., Saunders, R. C., Jones, D. W., Hommer, D., et al. (1998). In vivo association between alcohol intoxication, aggression, and serotonin transporter availability in nonhuman primates. American Journal of Psychiatry, 155, 1023-1028.

Heinz, A., Jones, D. W., Bissette, G., Hommer, D., Ragan, P., Knable, M., et al. (2002). Relationship between cortisol and serotonin metabolites and transporters in alcoholism. Pharmacopsychiatry, 35, 127-134.

Heinz, A., Jones, D. W., Mazzanti, C., Goldman, D., Ragan, P., Hommer, D., et al. (2000). A relationship between serotonin transporter genotype and in vivo protein expression and alcohol neurotoxicity. Biological Psychiatry, 47, 643-649.

Holmes, A., \& Hariri, A. R. (2003). The serotonin transporter gene-linked polymorphism and negative emotionality: Placing single gene effects in the context of genetic background and environment. Genes, Brain and Behavior, 2, 332-335.

Holmes, A., Lit, Q., Murphy, D. L., Gold, E., \& Crawley, J. N. (2003). Abnormal anxiety-related behavior in serotonin transporter null mutant mice: The influence of genetic background. Genes, Brain and Behavior, 2 , 365-380.

Katsuragi, S., Kunugi, H., Sano, A., Tsutsumi, T., Isogawa, K., Nanko, S., et al. (1999). Association between serotonin transporter gene polymorphism and anxietyrelated traits. Biological Psychiatry, 45, 368-370.

Kaufman, J., Yang, B. Z., Douglas-Palumberi, H., Houshyar, S., Lipschitz, D., Krystal, J. H., et al. (2004). Social supports and serotonin transporter gene moderate depression in maltreated children. Proceedings of the National Academy of Sciences of the United States of America, 101, 17316-17321.

Keightley, M. L., Winocur, G., Graham, S. J., Mayberg, H. S., Hevenor, S. J., \& Grady, C. L. (2003). An fMRI study investigating cognitive modulation of brain regions associated with emotional processing of visual stimuli. Neuropsychologia, 41, 585-596.

Kendler, K. S., Kuhn, J. W., Vittum, J., Prescott, C. A., \& Riley, B. (2005). The interaction of stressful life events and a serotonin transporter polymorphism in the prediction of episodes of major depression: A replication. Archives in General Psychiatry, 62, 529-535.

Kim-Cohen, J., Caspi, A., Moffitt, T. E., Harrington, H., Milne, B. J., \& Poulton, R. (2003). Prior juvenile diagnoses in adults with mental disorder: Developmental follow-back of a prospective-longitudinal cohort. Archives in General Psychiatry, 60, 709-717.

Kreek, M. J., \& Koob, G. F. (1998). Drug dependence: Stress and dysregulation of brain reward pathways. Drug and Alcohol Dependence, 51, 23-47.

LaForge, K. S., Yuferov, V., \& Kreek, M. J. (2000). Opioid receptor and peptide gene polymorphisms: Potential implications for addictions. European Journal of Pharmacology, 410, 249-268. 
Lander, E. S., Linton, L. M., Birren, B., Nusbaum, C., Zody, M. C., Baldwin, J., et al. (2001). Initial sequencing and analysis of the human genome. Nature, 409, 860-921.

Lange, K., Williams, L. M., Young, A. W., Bullmore, E. T., Brammer, M. J., Williams, S. C., et al. (2003). Task instructions modulate neural responses to fearful facial expressions. Biological Psychiatry, 53, 226-232.

LeDoux, J. E. (2000). Emotion circuits in the brain. Annual Review of Neuroscience, 23, 155-184.

Lesch, K. P., Bengel, D., Heils, A., Sabol, S. Z., Greenberg, B. D., Petri, S., et al. (1996). Association of anxiety-related traits with a polymorphism in the serotonin transporter gene regulatory region. Science, 274, 1527-1531.

Lesch, K. P., \& Mossner, R. (1998). Genetically driven variation in serotonin uptake: Is there a link to affective spectrum, neurodevelopmental, and neurodegenerative disorders? Biological Psychiatry, 44, 179-192.

Lewis, D. A. (1997). Development of the prefrontal cortex during adolescence: Insights into vulnerable neural circuits in schizophrenia. Neuropsychopharmacology, 16, 385-398.

Little, K. Y., McLaughlin, D. P., Zhang, L., Livermore, C. S., Dalack, G. W., McFinton, P. R., et al. (1998). Cocaine, ethanol, and genotype effects on human midbrain serotonin transporter binding sites and MRNA levels. American Journal of Psychiatry, 155, 207-213.

Lucki, I. (1998). The spectrum of behaviors influenced by serotonin. Biological Psychiatry, 44, 151-162.

Luthar, S. S., Cicchetti, D., \& Becker, B. (2000). The construct of resilience: A critical evaluation and guidelines for future work. Child Development, 71, 543-562.

Malhotra, A. K., \& Goldman, D. (1999). Benefits and pitfalls encountered in psychiatric genetic association studies. Biological Psychiatry, 45, 544-550.

Malison, R. T., Price, L. H., Berman, R., van Dyck, C. H., Pelton, G. H., Carpenter, L., et al. (1998). Reduced brain serotonin transporter availability in major depression as measured by [123I]-2 beta-carbomethoxy-3 beta-(4-iodophenyl)tropane and single photon emission computed tomography. Biological Psychiatry, 44, 1090-1098.

Mayberg, H. S. (2003a). Modulating dysfunctional limbiccortical circuits in depression: Towards development of brain-based algorithms for diagnosis and optimised treatment. British Medical Bulletin, 65, 193-207.

Mayberg, H. S. (2003b). Positron emission tomography imaging in depression: A neural systems perspective. Neuroimaging Clinics of North America, 13, 805-815.

Mazzanti, C. M., Lappalainen, J., Long, J. C., Bengel, D., Naukkarinen, H., Eggert, M., et al. (1998). Role of the serotonin transporter promoter polymorphism in anxiety-related traits. Archives in General Psychiatry, 55, 936-940.

Meaney, M. J., \& Szyf, M. (2005). Environmental programming of stress responses through DNA methylation: Life at the interface between a dynamic environment and a fixed genome. Dialogues in Clinical Neuroscience, 7, 103-123.

Melke, J., Landen, M., Baghei, F., Rosmond, R., Holm, G., Bjorntorp, P., et al. (2001). Serotonin transporter gene polymorphisms are associated with anxietyrelated personality traits in women. American Journal of Medical Genetics, 105, 458-463.

Meyer-Lindenberg, A., Hariri, A. R., Munoz, K. E., Mervis, C. B., Mattay, V. S., Morris, C. A., et al. (2005).
Neural correlates of genetically abnormal social cognition in williams syndrome. Nature Neuroscience, 8 , 991-993.

Moldin, S. O., \& Gottesman, II. (1997). At issue: Genes, experience, and chance in schizophrenia-Positioning for the 21st century. Schizophrenia Bulletin, 23, 547-561.

Moreno, F. A., Rowe, D. C., Kaiser, B., Chase, D., Michaels, T., Gelernter, J., et al. (2002). Association between a serotonin transporter promoter region polymorphism and mood response during tryptophan depletion. Molecular Psychiatry, 7, 213-216.

Munafo, M. R., Clark, T., \& Flint, J. (2005). Does measurement instrument moderate the association between the serotonin transporter gene and anxietyrelated personality traits? A meta-analysis. Molecular Psychiatry, 10, 415-419.

Murphy, D. L., Andrews, A. M., Wichems, C. H., Li, Q., Tohda, M., \& Greenberg, B. (1998). Brain serotonin neurotransmission: An overview and update with an emphasis on serotonin subsystem heterogeneity, multiple receptors, interactions with other neurotransmitter systems, and consequent implications for understanding the actions of serotonergic drugs. Journal of Clinical Psychiatry, 59(Suppl. 15), 4-12.

Nakamura, K., Kawashima, R., Nagumo, S., Ito, K., Sugiura, M., Kato, T., et al. (1998). Neuroanatomical correlates of the assessment of facial attractiveness. Neuroreport, 9, 753-757.

Narumoto, J., Yamada, H., Iidaka, T., Sadato, N., Fukui, K., Itoh, H., et al. (2000). Brain regions involved in verbal or non-verbal aspects of facial emotion recognition. Neuroreport, 11, 2571-2576.

Neumeister, A., Konstantinidis, A., Stastny, J., Schwarz, M. J., Vitouch, O., Willeit, M., et al. (2002). Association between serotonin transporter gene promoter polymorphism (5HTTLPR) and behavioral responses to tryptophan depletion in healthy women with and without family history of depression. Archives in General Psychiatry, 59, 613-620.

Patkar, A. A., Berrettini, W. H., Mannelli, P., Gopalakrishnan, R., Hoehe, M. R., Bilal, L., et al. (2004). Relationship between serotonin transporter gene polymorphisms and platelet serotonin transporter sites among African-American cocaine-dependent individuals and healthy volunteers. Psychiatry and Genetics, 14, 25-32.

Pezawas, L., Meyer-Lindenberg, A., Drabant, E. M., Verchinski, B. A., Munoz, K. E., Kolachana, B. S., et al. (2005). 5HTTLPR polymorphism impacts human cingulate-amygdala interactions: A genetic susceptibility mechanism for depression. Nature Neuroscience, 8, 828-834.

Phillips, M. L., Drevets, W. C., Rauch, S. L., \& Lane, R. (2003a). Neurobiology of emotion perception I: The neural basis of normal emotion perception. Biological Psychiatry, 54, 504-514.

Phillips, M. L., Drevets, W. C., Rauch, S. L., \& Lane, R. (2003b). Neurobiology of emotion perception II: Implications for major psychiatric disorders. Biological Psychiatry, 54, 515-528.

Plomin, R., Owen, M. J., \& McGuffin, P. (1994). The genetic basis of complex human behaviors. Science, 264, 1733-1739

Pohorecky, L. A. (1990). Interaction of ethanol and stress: Research with experimental animals-An update. Alcohol and Alcoholism, 25, 263-276.

Pohorecky, L. A. (1991). Stress and alcohol interaction: 
An update of human research. Alcoholism and Clinical Experimental Research, 15, 438-459.

Reif, A., \& Lesch, K. P. (2003). Toward a molecular architecture of personality. Behavioural Brain Research, 139, 1-20.

Rosenkranz, J. A., Moore, H., \& Grace, A. A. (2003). The prefrontal cortex regulates lateral amygdala neuronal plasticity and responses to previously conditioned stimuli. Journal of Neuroscience, 23, 11054-11064.

Rutter, M., Kim-Cohen, J., \& Maughan, B. (2006). Continuities and discontinuities in psychopathology between childhood and adult life. Journal of Child Psychology and Psychiatry, 47, 276-295.

Rutter, M., Moffitt, T. E., \& Caspi, A. (2006). Geneenvironment interplay and psychopathology: Multiple varieties but real effects. Journal of Child Psychology and Psychiatry and Allied Disciplines, 47, 226-261.

Sadikot, A. F., \& Parent, A. (1990). The monoaminergic innervation of the amygdala in the squirrel monkey: An immunohistochemical study. Neuroscience, 36, 431-447.

Schinka, J. A., Busch, R. M., \& Robichaux-Keene, N. (2004). A meta-analysis of the association between the serotonin transporter gene polymorphism (5HTTLPR) and trait anxiety. Molecular Psychiatry, 9, 197-202.

Schuckit, M. A. (1998). Biological, psychological and environmental predictors of the alcoholism risk: A longitudinal study. Journal of the Studies on Alcohol, 59, 485-494.

Schwartz, C. E., Wright, C. I., Shin, L. M., Kagan, J., \& Rauch, S. L. (2003). Inhibited and uninhibited infants "grown up": Adult amygdalar response to novelty. Science, 300, 1952-1953.

Scott, S., Knapp, M., Henderson, J., \& Maughan, B. (2001). Financial cost of social exclusion: Follow up study of antisocial children into adulthood. British Medical Journal, 323(7306), 191-195.

Sen, S., Burmeister, M., \& Ghosh, D. (2004). Metaanalysis of the association between a serotonin transporter promoter polymorphism (5-HTTLPR) and anxiety-related personality traits. American Journal of Medical Genetics, 127B, 85-89.

Shioe, K., Ichimiya, T., Suhara, T., Takano, A., Sudo, Y., Yasuno, F., et al. (2003). No association between geno- type of the promoter region of serotonin transporter gene and serotonin transporter binding in human brain measured by pet. Synapse, 48, 184-188.

Siegle, G. J., Steinhauer, S. R., Thase, M. E., Stenger, V. A., \& Carter, C. S. (2002). Can't shake that feeling: Event-related fMRI assessment of sustained amygdala activity in response to emotional information in depressed individuals. Biological Psychiatry, 51, 693-707.

Smith, H. R., Daunais, J. B., Nader, M. A., \& Porrino, L. J. (1999). Distribution of [3H]citalopram binding sites in the nonhuman primate brain. Annals of the New York Academy of Science, 877, 700-702.

Tessitore, A., Hariri, A. R., Fera, F., Smith, W. G., Chase, T. N., Hyde, T. M., et al. (2002). Dopamine modulates the response of the human amygdala: A study in Parkinson's disease. Journal of Neuroscience, 22, 9099-9103.

van Dyck, C. H., Malison, R. T., Staley, J. K., Jacobsen, L. K., Seibyl, J. P., Laruelle, M., et al. (2004). Central serotonin transporter availability measured with [123I]beta-CIT SPECT in relation to serotonin transporter genotype. American Journal of Psychiatry, 161, 525-531.

Venter, J. C., Adams, M. D., Myers, E. W., Li, P. W., Mural, R. J., Sutton, G. G., et al. (2001). The sequence of the human genome. Science, 291, 1304-1351.

Viding, E., Blair, R. J., Moffitt, T. E., \& Plomin, R. (2005). Evidence for substantial genetic risk for psychopathy in 7-year-olds. Journal of Child Psychology and Psychiatry and Allied Disciplines, 46, 592-597.

Wand, G. S., McCaul, M., Yang, X., Reynolds, J., Gotjen, D., Lee, S., et al. (2002). The mu-opioid receptor gene polymorphism (a118g) alters HPA axis activation induced by opioid receptor blockade. Neuropsychopharmacology, 26, 106-114.

Whale, R., Clifford, E. M., \& Cowen, P. J. (2000). Does mirtazapine enhance serotonergic neurotransmission in depressed patients? Psychopharmacology (Berlin), 148, 325-326.

Willeit, M., Praschak-Rieder, N., Neumeister, A., Pirker, W., Asenbaum, S., Vitouch, O., et al. (2000). [123I] beta-CIT SPECT imaging shows reduced brain serotonin transporter availability in drug-free depressed patients with seasonal affective disorder. Biological Psychiatry, 47, 482-489. 